\title{
Rules of chemokine receptor association with $T$ cell polarization in vivo
}

\author{
Chang H. Kim, ${ }^{1}$ Lusijah Rott,${ }^{1}$ Eric J. Kunkel, ${ }^{1}$ Mark C. Genovese, ${ }^{2}$ David P. Andrew, ${ }^{3}$ \\ Lijun Wu, ${ }^{3}$ and Eugene C. Butcher ${ }^{1}$
}
${ }^{1}$ Laboratory of Immunology and Vascular Biology, Department of Pathology, Stanford University School of Medicine, Stanford, California, USA, and Center for Molecular Biology and Medicine, Veterans Affairs Palo Alto Health Care System, Palo Alto, California, USA
${ }^{2}$ Division of Immunology and Rheumatology, Stanford University School of Medicine, Stanford, California, USA
${ }^{3}$ Millennium Pharmaceuticals Inc., Cambridge, Massachusetts, USA

Address correspondence to: Chang H. Kim, 3801 Miranda Avenue, Mail Code 154-B, VAMC Building 101, Room C4-111, Palo Alto, California 94304, USA. Phone: (650) 493-5000 ext. 63167; Fax: (650) 858-3986; E-mail: chkim@stanford.edu.

Current concepts of chemokine receptor (CKR) association with Th1 and Th2 cell polarization and effector function have largely ignored the diverse nature of effector and memory $T$ cells in vivo. Here, we systematically investigated the association of 11 CKRs, singly or in combination, with CD4 $\mathrm{T}$ cell polarization. We show that Th1, Th2, Th0, and nonpolarized T cells in blood and tissue can express any of the CKRs studied but that each CKR defines a characteristic pool of polarized and nonpolarized CD4 $\mathrm{T}$ cells. Certain combinations of CKRs define populations that are markedly enriched in major subsets of Th 1 versus Th 2 cells. For example, although Th0, Th1, and Th2 cells are each found among blood CD $4 \mathrm{~T}$ cells coordinately expressing CXCR3 and CCR4, Th1 but not Th2 cells can be $\mathrm{CXCR}^{+} \mathrm{CCR} 4^{-}$, and $\mathrm{Th} 2$ but only rare Th 1 cells are CCR $4^{+} \mathrm{CXCR} 3^{-}$. Contrary to recent reports, although CCR7- cells contain a higher frequency of polarized CD4 T cells, most Th1 and Th2 effector cells are $\mathrm{CCR} 7^{+}$and thus may be capable of lymphoid organ homing. Interestingly, Th1-associated CKRs show little or no preference for Th1 cells except when they are coexpressed with CXCR3. We conclude that the combinatorial expression of CKRs, which allow tissue- and subset-dependent targeting of effector cells during chemotactic navigation, defines physiologically significant subsets of polarized and nonpolarized $\mathrm{T}$ cells.

J. Clin. Invest. 108:1331-1339 (2001). DOI:10.1172/JCI200113543.

\section{Introduction}

Chemokines and chemokine receptors (CKRs) play critical roles in homeostatic and inflammatory migration of diverse cell types including memory and effector $\mathrm{T}$ cells (1-4). Chemokines are expressed by specific cell types in microenvironments, forming chemokine- or chemoattractant-defined microdomains. Chemokines induce firm adhesion of rolling leukocytes and are thought to help direct subsequent transmigration through endothelial cells into surrounding tissues. Subsequently, cells can migrate sequentially to one chemoattractant source after another in a process of multistep navigation that targets subsets to appropriate microenvironments as a function of their patterns of CKR expression. This step-by-step navigation allows lymphocyte subset localization to be determined by the combination of CKRs they express (5).

Among the diverse types of effector $T$ cells that are generated from naive $T$ cells, two distinct types of T cells (type 1 and 2 , or Th1 and Th2, specifically for polarized CD4 T cells) in particular are distinguished based upon their effector cytokine production. Th2 cells produce IL-4, IL-5, IL-6, and/or IL-13, while Th1 cells produce IFN- $\gamma(6-8)$. Polarized T cells are involved in specific effector functions and in progression of many diseases that also display strikingly polarized pathological features. One the other hand, the majori- ty (on average 77\%) of memory CD4 T cells in human blood are unable to produce the Th1 or Th2 cytokines and thus have been classified as nonpolarized. These nonpolarized CD4 $\mathrm{T}$ cells (Tnp's) may comprise functionally specialized subsets themselves (9-12).

A number of groups have reported that Th1 and Th2 cells express distinct sets of CKRs: Th1 cells preferentially express CCR5 and CXCR3 (Th1-asssociated CKRs), while Th2 cells preferentially express CCR3, CCR4, and CCR8 (Th2-associated CKRs) (13-19). With other, previous reports on differential roles of adhesion molecules $(20,21)$, these studies on CKRs led to a proposal that Th1 and Th 2 cells have homing potentials quite different from each other. The existence of characteristic Th1 versus Th2 homing properties would be attractive, because it might provide a mechanism for amplification of Th1- or Th2-associated inflammation at tissue sites of appropriate chemokine expression. However, these studies primarily have relied on artificial in vitro regimens to induce $T$ cell polarization, and the extent to which different CKRs correlate with Th1 and Th2 cell differentiation in physiologic settings has been controversial (22-27). Moreover, given the requirement for trafficking of effector cells to diverse tissue sites in the body, it seems likely that substantial heterogeneity must exist within both polarized and 
nonpolarized subsets, as for other memory/effector cell subsets. Thus, we postulated that CKR expression patterns of polarized and nonpolarized cells must be complex and heterogeneous to allow their differential tissue trafficking in vivo. At the same time, we were interested to determine whether, within this heterogeneity, certain patterns or combinations of CKR expression might distinguish major Th1, Th2, or Tnp cell subsets. To address these issues we have systematically evaluated patterns of CKR expression by polarized and nonpolarized $\mathrm{T}$ cell populations in human blood. In contrast to previous reports, our results reveal a remarkable heterogeneity of CKR expression by circulating and tissue Th1, Th2, Th0, and Tnp memory $\mathrm{T}$ cells and show that each of the CKRs studied can be expressed by cells of any functional subset. Nevertheless, we show that each CKR-expressing memory $T$ cell population has a characteristic composition of polarized and nonpolarized memory cells and that certain patterns of overlapping CKR expression define populations highly enriched in Th1 versus Th2 cells. Such patterns of overlapping CKR expression may allow differential targeting of effector cells during chemotactic navigation.

\section{Methods}

$A b$ 's. Ab's to CD4 (RPA-T4), CD45RA (HI100), CD45RO (UCHL1), and IFN- $\gamma$ (4S.B3) were purchased from PharMingen (San Diego, California, USA). Anti-IL-4-phycoerythrin (anti-IL-4-PE; 3010.211) was from Becton Dickinson Immunocytometry Systems (Mountain View, California, USA). Ab's to CXCR6 (56811.111), CCR1 (53504.111), CCR2 (48607.121), CCR5 (45549.111), CCR6 (53103.111), CXCR3 (49801.111), and CXCR5 (51505.111) were from R\&D Systems Inc. (Minneapolis, Minnesota, USA). Ab's to CCR3 (7B11), CCR4 (1G1), CCR7 (7H12-12-2), and CCR9 (GPR96-1) were from Millennium Pharmaceuticals (Boston, Massachusetts, USA

Cell isolation. Human peripheral blood was obtained from Stanford University Blood Center (Stanford, California, USA), and PBMCs were isolated by using a density gradient centrifuge on Histopaque-1077 (Sigma Chemical Co., St. Louis, Missouri, USA). CD4 ${ }^{+} \mathrm{T}$ cells (purity $>97 \%$ ) were isolated by depleting non-CD4 ${ }^{+}$ $\mathrm{T}$ cells using a magnetic bead depletion method (Miltenyi Biotec, Auburn, California, USA). Isolated CD4 $T$ cells were stained with appropriate Ab's and used for cytokine analysis. After CD4 T cells were stained with two different CKRs (CXCR3-FITC and anti-CCR2, CCR4, -CCR5, -CCR6, or -CCR7-CyChrome, or CCR7FITC and anti-CCR4-CyChrome), single- and doublepositive populations were sorted by FACSVantage SE (Becton Dickinson Immunocytometry Systems, San Jose, California, USA).

Synovial fluid was obtained from psoriatic arthritis patients undergoing diagnostic arthrocentesis. Diluted synovial fluid (1:2 in PBS) was centrifuged at $250 \mathrm{~g}$ before being layered over Ficoll (Amersham Pharmacia Biotech,
Piscataway, New Jersey, USA) to isolate the mononuclear layer. All human subject protocols were approved by the Institutional Review Board at Stanford University.

Cytokine single cell analysis of CKR-expressing CD4 T cells. CD4 T cells were prestained with Ab's (anti-CKR and anti-CD45RA or anti-cutaneous lymphocyte-associated antigen) and activated for 4 hours with PMA (50 $\mathrm{ng} / \mathrm{ml})$ and ionomycin $(1 \mu \mathrm{M})$ in the presence of monensin $(10 \mu \mathrm{g} / \mathrm{ml}$; Sigma Chemical Co.). This prestaining method yielded similar results to the experiments using sorted $T$ cell subsets. Importantly, the staining patterns of CKRs allophycocyani (APC) cutaneous lymphocyte-associated antigen (CLA; FITC), and CD45RA (CyChrome) were preserved after activation in this condition. Activated cells were fixed and permeabilized using Cytofix/Cytoperm solution (PharMingen) and stained with appropriate isotype control Ab's or anti-IL-4-PE and anti-IFN- $\gamma$-APC. Simultaneous staining for IL-4, IFN- $\gamma$, and CKRs allowed characterization of the frequency of Th1 cells, memory/effector CD4 cells producing only IFN- $\gamma$; Th 2 cells, producing only IL-4; Th0 cells producing both IFN- $\gamma$ and IL-4; and Tnp's producing neither IFN- $\gamma$ nor IL-4 among CD4 $T$ cells expressing each CKR. Data were analyzed on FACScalibur using CellQuest program (Becton Dickinson Immunocytometry Systems). For analysis of Th1 and Th 2 content in $\mathrm{CCR}^{+/-}, \mathrm{CXCR}^{+/-} \mathrm{CCR} 4^{+/-}$, or $\mathrm{CXCR}^{+/-} \mathrm{CCR}^{+/-}, \mathrm{CXCR}^{+/-} \mathrm{CCR} 2^{+/-}, \mathrm{CXCR}^{+/-}$ $\mathrm{CCR}^{+/-}$, or $\mathrm{CXCR}^{+/-}{ }^{+-} \mathrm{CXCR} 5^{+/-} \mathrm{CD} 4 \mathrm{~T}$ memory cell subsets, cells were sorted and then analyzed for expression of IL-4 and IFN- $\gamma$.

Statistics. To calculate significant differences between two groups, two-tailed and paired Student $t$ test was used.

\section{Results}

Each CKR-defined memory T cell population has a characteristic composition of polarized and nonpolarized subsets. Initially, we investigated the production of Th1 and Th2 cytokines by circulating fresh blood CD4 $\mathrm{T}$ cell subsets expressing different CKRs. Expression of CKRs on CD4 $T$ cells characterizes distinct populations of $T$ helper cells. Many members of the CKR family such as CCR2 (28), CCR3 (29-31), CCR4 $(22,32)$, CCR5 $(33,34)$, CCR6 (35-37), CXCR3 (38), CXCR5 (39), and CXCR6 $(19,40,41)$ are expressed only on memory (CD45RAor CD45RO $\mathrm{R}^{+}$CD4 $\mathrm{T}$ cells (Figure 1a). CCR7 (42) and CCR9 (43-45) are expressed on both naive and memory CD4 T cells (Figure 1a). CCR1 and CXCR4 are not included in this study, since they are expressed by too few (CCR1) or by almost all (CXCR4) blood CD4 T cells (data not shown). Each CKR-expressing memory CD4 $T$ cell population is composed of characteristic frequencies of Th1, Th2, Th0, and Tnp cells (Figure 1, b and c). CXCR3-, CCR5-, and CXCR6-expressing CD4 $\mathrm{T}$ cells are enriched for Th1 cells $(45-49 \%, n=8)$, although cells expressing these CKRs also contain significant numbers of Th 2 cells (1-3\% of each population; $30-90 \%$ of the Th2 cell frequency in the whole memory population). $\mathrm{CCR} 2{ }^{+} \mathrm{CD} 4 \mathrm{~T}$ cells, while rela- 
a

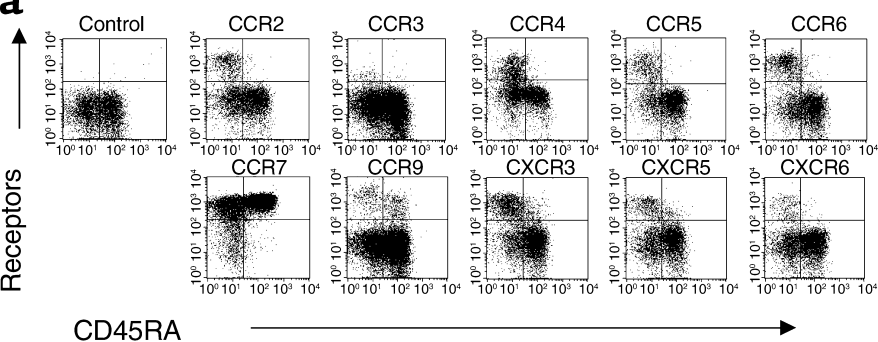

b
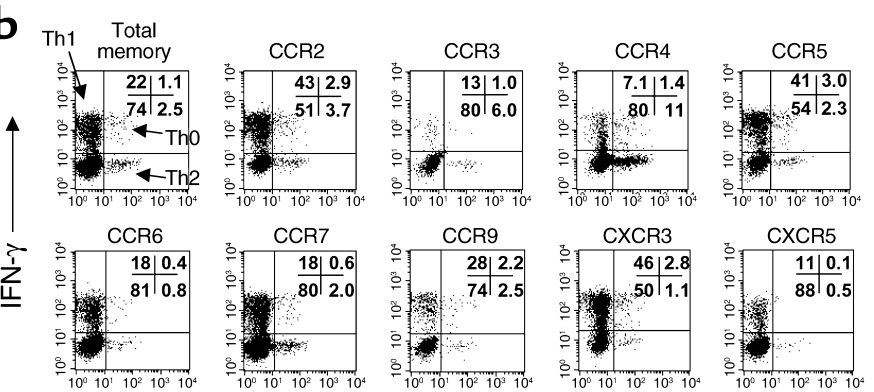

IL-4

C

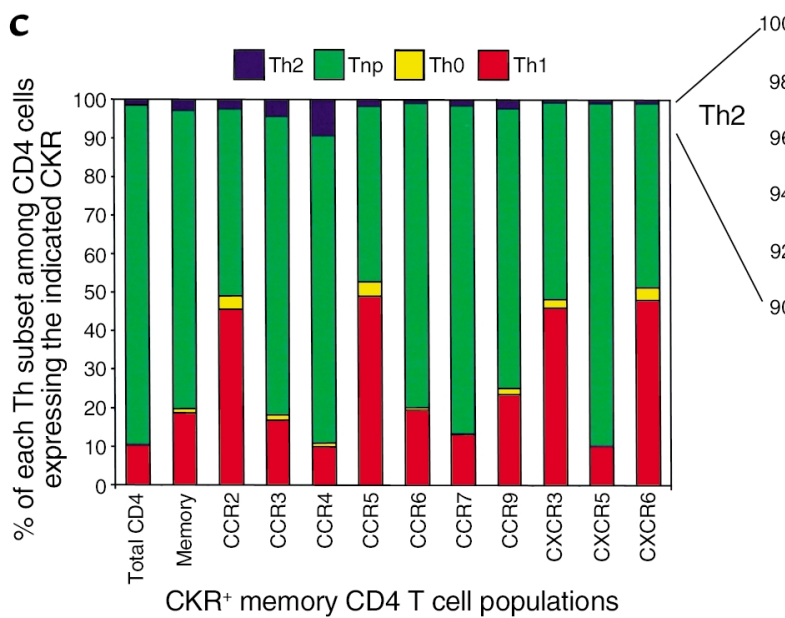

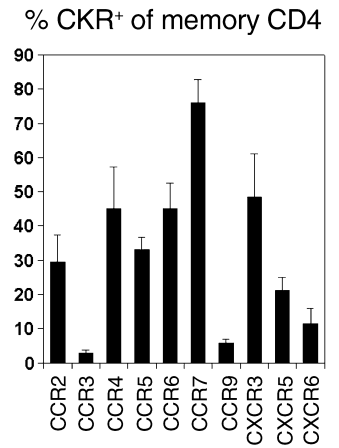
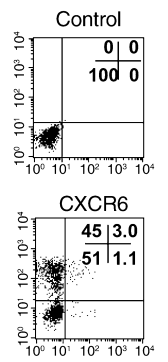

$\longrightarrow$
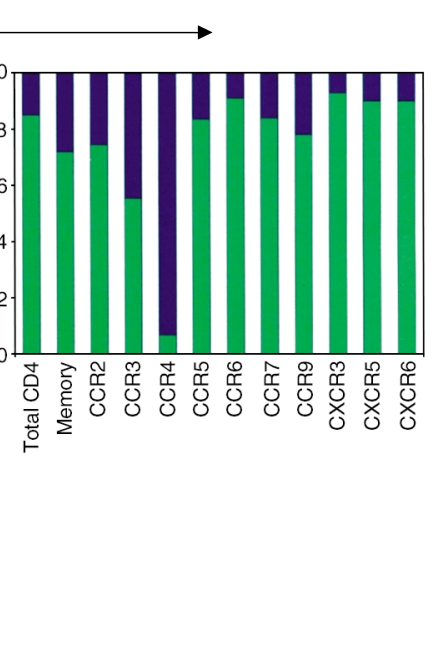

\section{Figure 1}

Profiles of polarized and nonpolarized $\mathrm{T}$ cell frequencies in $\mathrm{CKR}^{+}$ memory CD4 T cell populations. (a) Expression of CKRs on total (left, FACS plots) and memory CD4 T cells (right, histogram). Frequencies of Th1 (IFN- $\gamma^{+}$IL-4- $)$, Th2 (IL-4+ IFN- $\gamma)$, Th0 (IFN- $\gamma^{+}$ IL-4 $4^{+}$, or Tnp (IFN- $\gamma$ IL-4- $)$ cells in total, memory, or CKRexpressing memory CD4 $\mathrm{T}$ cell populations are shown as dot plots (b) and graphs (c). Peripheral blood CD4 T cells were activated by PMA and ionomycin for 4 hours before cytokine analysis. Each $\mathrm{CKR}^{+/-}$population is gated (b and $\mathbf{c}$ ). Averages from 6-11 different donors are shown. tively enriched for Th1 cells, contain similar levels of Th2 cells to the total memory CD $4 \mathrm{~T}$ cell pool. CCR4 ${ }^{+}$ cells are enriched for Th2 cells $(9.3 \%)$ compared with total memory CD4 $\mathrm{T}$ cells (2.8\%). Surprisingly, although Th1 cells are relatively depleted among CCR $4^{+}$cells, in fact approximately $10 \%$ of CCR $4^{+}$cells are Th1, as well. CCR $3^{+}$cells, a rare subset in blood, contain twice the frequency of Th2 cells as the bulk memory CD 4 cell population, but on average contain a similar frequency of Th 1 cells. Th0 cells are enriched within CCR2-, CCR5-, CXCR3-, and CXCR6-expressing memory CD4 T cells (Figure 1c).

The majority $(77 \%, n=9)$ of blood memory CD4 T cells are nonpolarized Tnp's, unable to produce IL-4 or IFN- $\gamma$. Eighty-eight percent of $\mathrm{CXCR}^{+}$or $84 \%$ of CCR7 ${ }^{+}$ T cells are Tnp's $(n=8)$, levels higher $(77 \%)$ than that of total memory T cells (Figure 1c). Correlating with their reduced numbers of Th1 cells, CCR $4^{+} \mathrm{T}$ cells also contain many $(81 \%)$ Tnp's. Fewer but still considerable numbers of Tnp's are contained within the CCR2-positive
(46\%), CCR5-positive (45\%), CXCR3-positive (48\%), and CXCR6-positive (48\%) populations. CCR3-, CCR6-, and CCR9-positive subsets contain similar frequencies of Tnp's to the total memory CD4 T cells.

CKR expression profiles of Th1, Th2, Th0, and Tnp cells. Precommitted Th1 and (especially) Th2 cells represent only a small fraction of most of these CKR-defined subsets. To characterize polarized effector and nonpolarized memory cells more directly, we therefore identified these minor effector cell populations based on cytokine staining and directly evaluated their expression of the different receptors. Most Th1 cells express CXCR3 (on average $90 \%$ ) (Figure 2 ). In contrast, only approximately $50 \%$ of Th 1 cells express CCR5, only approximately $15 \%$ of Th1 cells express CXCR6, and only approximately $42 \%$ express CCR2. Almost all ( 95\%) Th2 cells express CCR4, while CCR3 marks only approximately $3 \%$ of Th 2 cells. Many Th0 cells express CXCR3, CCR 5 , CCR2, CCR4, and CCR7 receptors that are expressed by many Th 1 and/or Th 2 cells. CCR 6 is also expressed 

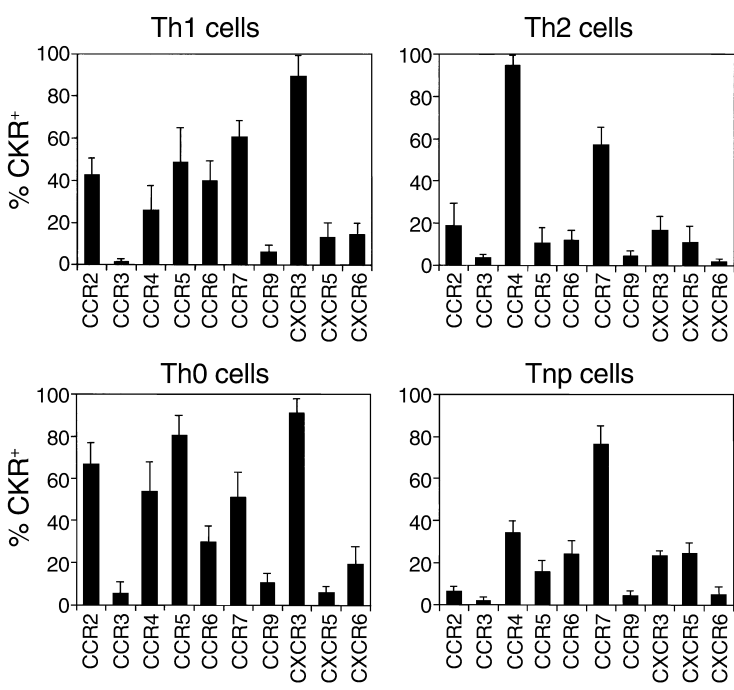

\section{Figure 2}

CKR expression by Th1, Th2, Th0, and Tnp cell populations. Percentage of peripheral blood Th1, Th2, Th0, and nonpolarized cells expressing each CKR was examined by gating on Th1 (IFN- $\gamma^{+}$IL-4- $4^{-}$, Th2 (IL-4+ IFN- $\gamma$ ), Th0 (IFN- $\gamma^{+}$IL-4 $\left.4^{+}\right)$, or Tnp (IFN- $\gamma$ IL-4- $)$ cells. Averages from at least six different donors with SDs.

on approximately $40 \%$ of Th1 and approximately $10 \%$ of Th2 cells. Many Tnp's express CCR7 (76\%), CCR4 (34\%), CXCR5 (25\%), CXCR3 (24\%), and CCR6 (24\%) in a decreasing order. Notably, CXCR5 is particularly expressed by more Tnp versus Th1, Th2, or Th0 cells. In contrast, $\mathrm{CCR} 2^{+} \mathrm{T}$ cells are underrepresented in the Tnp versus Th1, Th2, or Th0 cell population.

The majority of polarized effector T cells are CCR $7^{+}$. A CRK CCR7 has been described previously as a defining factor for two different memory $\mathrm{T}$ cell populations: $\mathrm{CCR} 7^{+}$memory cells were associated with a proliferation reserve population and lacked effector function, while CCR7- cells were equated with immediate effector $\mathrm{T}$ cells that produce effector cytokines upon stimulation (42). However, our results show that both CCR7 ${ }^{+}$ and CCR7- $\mathrm{T}$ cells contain effector $\mathrm{T}$ cells and that there are in fact many more $\mathrm{CCR}^{+}$than $\mathrm{CCR}^{-}$polarized $\mathrm{T}$ cells in human blood (Figure 3 ). Approximately, $30 \%$ of CCR7 $7^{-}$memory $\mathrm{T}$ cells and $15 \%$ of CCR7 ${ }^{+}$memory $\mathrm{T}$ cells were IFN- $\gamma$ producers, and $6 \%$ of CCR7 ${ }^{-}$and $3 \%$ of CCR7 ${ }^{+}$memory T cells were IL-4 producers (Figure 3a). In blood, CCR7- memory $\mathrm{T}$ cells are relatively rare ( $24 \%$ of memory CD $4 \mathrm{~T}$ cells) when compared with CCR $7^{+}$memory $\mathrm{T}$ cells $(76 \%$ of memory CD $4 \mathrm{~T}$ cells) (Figure 1a). Therefore, when the absolute number of polarized effector (IL-4 or IFN- $\gamma$ producers) T cells was calculated based on the frequency of each memory subset in blood $\left(42 \% \pm 8 \%\right.$ of CD 4 cells are CCR7 ${ }^{+}$, $11 \% \pm 3 \%$ are CCR7 $-; n=8)$, there are more CCR7 ${ }^{+}$ IFN- $\gamma$ ( $8 \%$ of CD4 T cells) than CCR7- IFN- $\gamma(3.5 \%)$ producers among CD4 T cells (Figure 3b). Similarly, more $\mathrm{CCR}^{+}(1.6 \%$ of CD4 T cells) than CCR7- $(0.7 \%)$ IL-4 producers are present in the circulation.
We also examined the effector frequencies of $\mathrm{CXCR}^{+/-}$memory CD4 $\mathrm{T}$ cell populations for direct comparison with the $\mathrm{CCR} 7^{+/-}$populations. $\mathrm{CXCR5}^{+}$ cells are a subset of the CCR $7^{+}$population (on average, $26 \%$ of $\mathrm{CCR}^{+}$memory $\mathrm{T}$ cells are $\mathrm{CXCR} 5^{+}$, whereas almost no CCR7 ${ }^{-} \mathrm{T}$ cells express CXCR5; not shown), and $\mathrm{CXCR} 5^{+}$cells are largely defective in production of IL-4 and IFN- $\gamma(9,10)$. Fewer polarized effectors were found among $\mathrm{CXCR}^{+}$than among $\mathrm{CXCR}^{-}$or $\mathrm{CCR}^{+/}-$ memory $\mathrm{T}$ cell populations. When the absolute number of polarized cells in blood was examined, most effectors were found in CXCR5- but not in CXCR5 $5^{+}$memory $\mathrm{T}$ cell population, in contrast to the distribution of polarized cells in $\mathrm{CCR} 7^{+-}$populations (Figure 3 ). These data suggest (a) that not all, but a subset of the CCR7 ${ }^{+}$memory $\mathrm{T}$ cell population (e.g., CXCR5 ${ }^{+} \mathrm{T}$ cells), are really defective in Th1/2 effector cytokine production and (b) that the majority of effector $\mathrm{T}$ cells are $\mathrm{CCR} 7^{+}$. Therefore, many polarized cells may circulate through central lymphoid tissues as well as tissue sites of inflammation, but these IFN- $\gamma$ - and IL-4-producing cells are largely independent of the presumed $\mathrm{B}$ helper-related $\mathrm{T}$ cells expressing the follicle homing-associated CKR CXCR5.

More specific definition of polarized T cells by combinations of CKRs. Leukocyte microenvironmental homing is controlled not by individual receptors, but rather through multistep processes of navigation that require sequential engagement of several chemoattractant (and other)
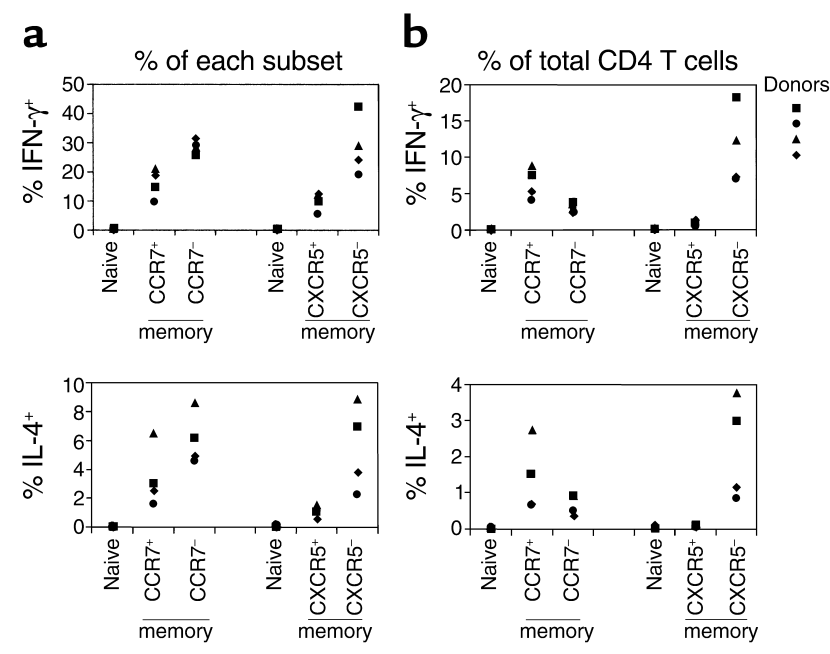

Figure 3

Polarized T cell populations are enriched in frequency among CCR7T cells, but are predominantly $C C R 7^{+}$. (a) The frequencies of IL-4- or IFN- $\gamma$-producing cells are shown as percentage of each CKR-positive or -negative subset by gating on naive $\left(C D 45 \mathrm{RA}^{+}\right), \mathrm{CCR} 7^{+}, \mathrm{CCR} 7^{-}$, $\mathrm{CXCR}^{+}$, or $\mathrm{CXCR} 5^{-} \mathrm{CD} 45 \mathrm{RO}^{+} \mathrm{CD} 4 \mathrm{~T}$ cell population. (b) The absolute frequency (\%) of IL-4- or IFN- $\gamma$-producing cells among $\mathrm{CCR}^{+/-}$or $\mathrm{CXCR}^{+/-} \mathrm{CD}_{5} 5 \mathrm{RO}^{+}$subsets in blood CD4 T cells. The absolute frequency (\%) was obtained by normalizing (i.e., multiplying) the frequencies of IL-4- or IFN- $\gamma$-producing cells in each subset (the values in the a) by the frequency of each memory subset in total CD4 T cells ( 0.42 for $\mathrm{CCR}^{+} ; 0.11$ for $\mathrm{CCR}^{-} ; 0.1$ for $\mathrm{CXCR}^{+} ; 0.43$ for CXCR5 $; n=8)$. CD4 cells $\left(5 \times 10^{4}\right)$ were acquired for each analysis. Results from four donors are shown. 

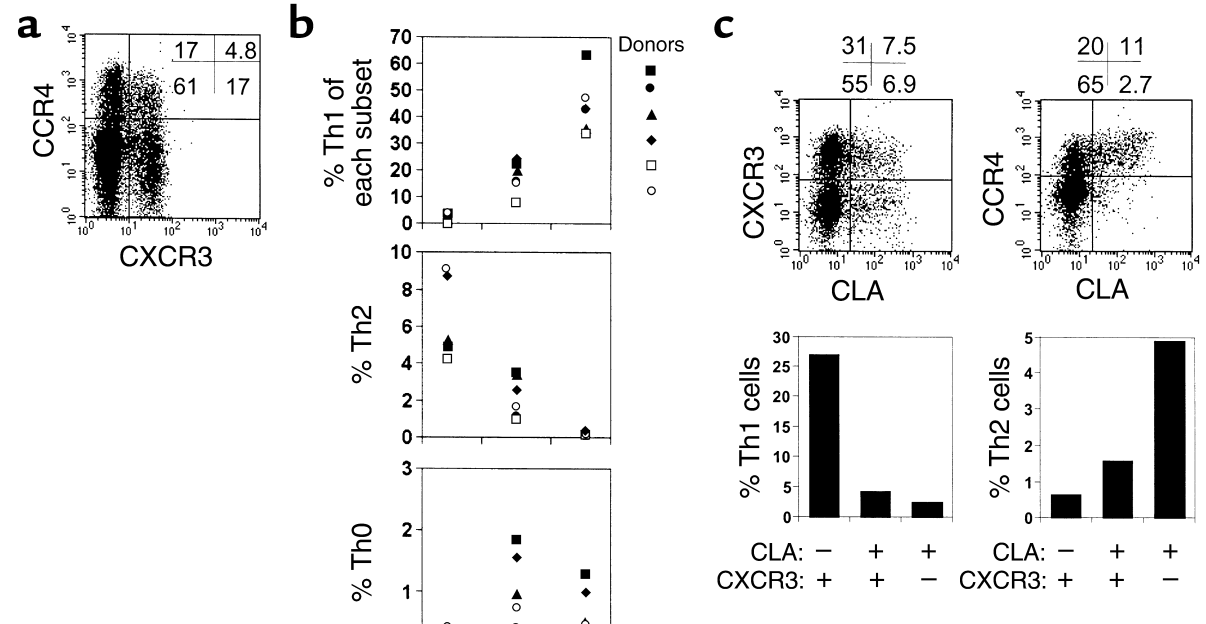

\begin{abstract}
Figure 4
Polarized CD4 $T$ cell populations defined by CXCR3 and CCR4 in combination. (a) Coexpression of CXCR3 and CCR4, and (b) frequencies of Th1, Th2, and Th0 cells in $\mathrm{CCR}^{+}{ }^{+} \mathrm{CXCR}^{-}, \mathrm{CCR}^{+}{ }^{+} \mathrm{CXCR}_{3}{ }^{+}$, and $\mathrm{CCR}^{-}{ }^{-} \mathrm{CXCR} 3^{+}$populations. Each subset was individually sorted from peripheral blood CD4 T cells for cytokine analyses. (c) Expression of CXCR3 and CCR4 and frequencies of Th1 and Th 2 cells in the CLA ${ }^{+/-}$(skinhoming receptor) memory CD4 T cell subsets. Representative of four independent experiments.
\end{abstract}

receptors expressed in combination on the cell surface (5). We therefore asked if overlapping patterns of expression of CKRs (especially CXCR3 and CCR4, which are expressed by most Th1 or Th 2 cells, respectively, as shown in Figure 2) might more effectively distinguish Th1 versus Th2 cells. To ask whether singly positive or coexpressing subsets display distinctive $\mathrm{Th}$ effector associations, we separated the CCR $4^{+} \mathrm{CXCR} 3^{-}$, $\mathrm{CCR}^{+}{ }^{+} \mathrm{CXCR}^{+}$, and $\mathrm{CCR} 4-\mathrm{CXCR}^{+}{ }^{+}$populations and examined the polarized $T$ helper subset composition of each population (Figure 4). Surprisingly, Th1 cells (representing $37-65 \%$ ) but almost no Th2 cells are found in the CCR4 ${ }^{-} \mathrm{CXCR} 3^{+}$population, while Th2 (up to $10 \%$ ), but very few Th 1 cells $(<2 \%)$ are CCR $4^{+}$CXCR $3^{-}$. Interestingly, the double-positive CCR $4^{+} \mathrm{CXCR} 3^{+}$cells have intermediate levels of Th1 (10-20\%) and Th2 ( 2\%) cells and contain more Th0 cells (up to $2 \%$ ) than the other two subsets (Figure 4b). On average, $88 \%$ of Th1 cells in blood are in fact $\mathrm{CXCR}^{+}{ }^{+} \mathrm{CCR} 4^{-}$, and $90 \%$ of Th2 cells are CXCR3-CCR4+, so that these combinations of receptors define major subsets of circulating effector-polarized $T$ cells. Thus, analyses of these receptors in combination reveal a more specific phenotype for most Th1 (CXCR3 ${ }^{+}$CCR4-, $88 \%$ of Th1) and Th2 (CXCR3-CCR4 ${ }^{+}, 90 \%$ of $\mathrm{Th} 2$ ) cells and show that $\mathrm{CXCR}^{+} \mathrm{Th} 2$ cells and CCR $4^{+}$Th 1 cells are primarily within a $\mathrm{CXCR} 3^{+} \mathrm{CCR} 4^{+}$double-positive population, which contains a mixture of Th0, Th 1 , and Th 2 cells.

In the context of the multistep processes of cell migration, individual receptors can play different roles in different physiological settings (at different steps). As an example, CCR4 has been implicated in apparently "conflicting" roles as (a) a preferential Th2 cell chemoattractant receptor, and (b) a lymphocyte "homing receptor" for skin involved in vascular recognition during lymphocyte (presumably both Th1 and Th2) recruitment from the blood in diverse settings of cutaneous inflammation (46). To determine if overlapping receptor patterns could help explain this apparent conflict, we analyzed the phenotype of skin-homing memory $\mathrm{T}$ cells defined by expression of the CLA. As shown in Figure $4 c$, many $\mathrm{CLA}^{+} \mathrm{CD} 4 \mathrm{~T}$ cells coordinately express CXCR3 and CCR4: as reported previously, almost all $\mathrm{CLA}^{+} \mathrm{T}$ cells are CCR4 $4^{+}(46)$, but fully half of $\mathrm{CLA}^{+} \mathrm{T}$ cells are also $\mathrm{CXCR}^{+}$(Figure 4c). Interestingly, compared with CLA-CXCR3 ${ }^{+} \mathrm{CD} 4 \mathrm{~T}$ cells (most of which are CCR4-), $\mathrm{CLA}^{+} \mathrm{CXCR}^{+} \mathrm{T}$ cells (which are almost all $\mathrm{CCR} 4^{+}$) contain reduced numbers of Th1 cells, consistent with the reduction frequency of Th1 cells among CXCR3 cells coexpressing CCR4. The frequency of Th2 cells is also lower among $\mathrm{CLA}^{+} \mathrm{CXCR}^{+}$than among $\mathrm{CLA}^{+}$CXCR3- cells (even though both populations are predominantly CCR $4^{+}$). We conclude that many skinhoming T cells display both CXCR3 and CCR4 and that the frequencies of Th1 and Th2 cells in each subset closely correlate with the coexpression pattern of the two receptors as predicted from Figure $4 a$ and $b$.

CKR association with T cell polarization in inflamed tissues. It is important to ask whether the same rules of CKR association with polarization of circulating blood CD4 $T$ cells would apply for the $T$ cells in extralymphoid tissues, particularly inflamed tissues. To answer this, we examined CKR expression by polarized and nonpolarized $T$ cells in synovial fluids from patients with psoriatic arthritis, a type 1 polarized disease (47). All of the arthritis synovial CD4 $\mathrm{T}$ cells were memory cells (CD45RA-CD45RO ${ }^{+}$; Figure 5b). These CD4 T cells differ from blood $\mathrm{T}$ cells in that many more express the CKR CCR5 (71-89\%), CXCR3 (68-85\%), CXCR6 (22-61\%), and CCR2 (33-47\%) ( $n=3$, Figure 5a). Surprisingly, approximately half (48.5\%) of the tissue CD4 T cells also express CCR4, a pro-Th2 receptor. Most of these $\mathrm{CCR} 4^{+} \mathrm{T}$ cells coexpress CXCR3 (Figure 5c). Furthermore, one-third of the synovial CD4 T cells also express CCR7, a CKR proposed by others to define lymphoid tissue-homing lymphocytes excluded from extralymphoid tissues. Small but significant percentages of the CD4 $\mathrm{T}$ cells express CXCR5 (4-9.5\%) or 
a

Arthritis
synovial fluid CD4 T cells

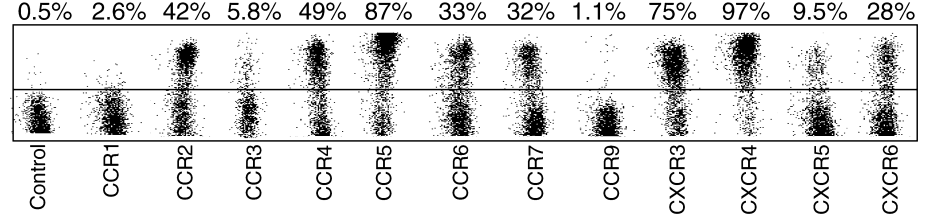

b

d

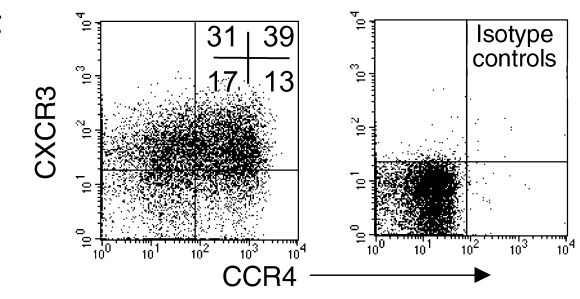

e
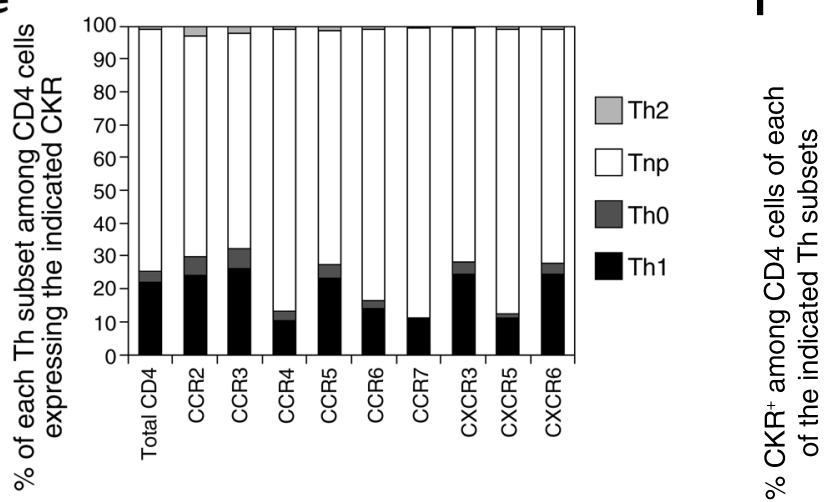

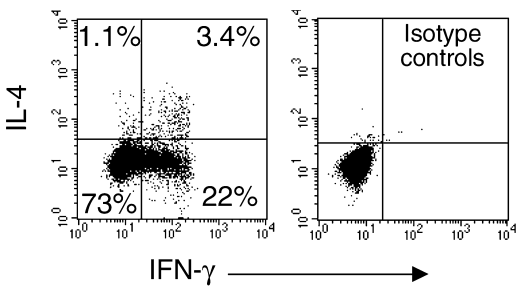

f
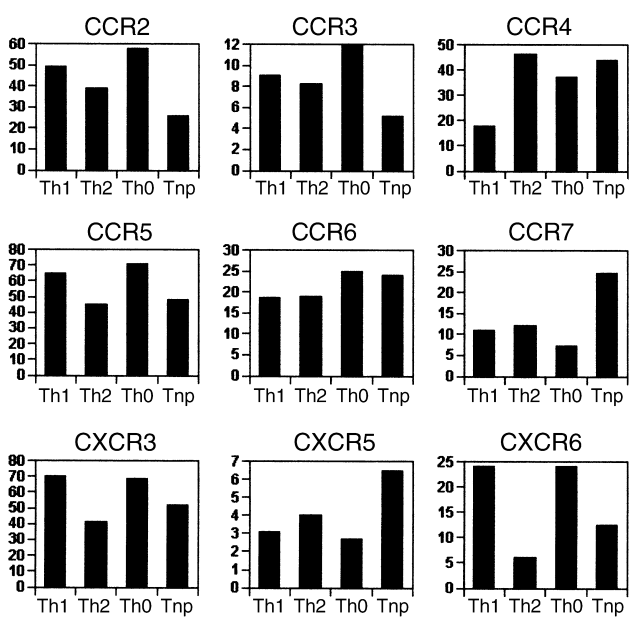

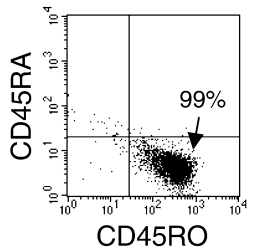

CD45RO

\section{Figure 5}

CKR association with T cell polarization in inflammatory tissues (CD4 T cells in psoriatic arthritic synovial fluid). (a) CKR expression by synovial CD4 T cells. (b) CD45RA and CD45RO expression by synovial CD4 T cells. (c) Coexpression of CXCR3 and CCR4 by synovial fluid CD4 T cells. (d) Intracellular IFN- $\gamma$ and IL-4 production pattern of arthritic synovial CD4 T cells. (e) Frequencies of Th1 (IFN- $\gamma^{+}$IL-4-), Th2 (IL-4 IFN- $\gamma$ ), Th0 (IFN- $\gamma^{+}$IL-4 $4^{+}$), or Tnp (IFN- $\gamma^{-}$IL-4- $)$cells in CKR-expressing CD4 T cell populations are shown. (f) Expression of CKRs by Th1, Th2, Th0, and Tnp cells in arthritis synovial fluid. Prestained cells with anti-CKR and anti-CD4 were activated by PMA and ionomycin for 4 hours in the presence of monensin before intracellular staining of IFN- $\gamma$ and IL-4. Representative data from three different experiments are shown.

CCR3 (5-8\%). CCR1 $1^{+}$or CCR9 ${ }^{+}$CD4 $\mathrm{T}$ cells are relatively rare $(0.5-2.1 \%)$ in synovial fluid.

We next examined the frequency of polarized and nonpolarized $\mathrm{T}$ cells in $\mathrm{CKR}^{+} \mathrm{CD} 4 \mathrm{~T}$ cell populations in synovial fluid. Many more Th1 and Th0, but fewer Th2, cells are found in arthritic synovial fluid than in blood, confirming that they are mainly type 1 polarized (Figure 5d). $\mathrm{CCR}^{+}, \mathrm{CCR}^{+}, \mathrm{CCR}^{+}, \mathrm{CXCR}^{+}$, and $\mathrm{CXCR}^{+} \mathrm{CD} 4 \mathrm{~T}$ cell populations contain slightly more than or similar frequencies of Th 1 and Th 0 cells to the total $\mathrm{CD} 4 \mathrm{~T}$ population, while $\mathrm{CCR} 4^{+}, \mathrm{CCR}^{+}$, $\mathrm{CXCR}^{+}$, and CCR $6^{+}$populations contain reduced, but significant, numbers of Th 1 cells. Synovial CCR $4^{+} \mathrm{T}$ cells do not contain more Th2 cells than other $\mathrm{CKR}^{+}$ subsets (Figure 5e). Expression of CKRs by Th1, Th2, Th0, and Tnp cells are shown in Figure 5f. Although expressed by all subsets, CCR 4 is expressed by more Th2, Tnp, and Th0 than Th1 cells. In contrast, CXCR6, CXCR3, CCR5, and CCR2 are expressed by more Th1 and Th0 than Th2 and Tnp cells. As in blood, CXCR5 and CCR7 are expressed by more Tnp than Th1, Th2, and Th0 cells in synovial fluid.

Coexpression of CXCR 3 confers polarity toward Th1 on CKR ${ }^{+}$ populations. To explore further the significance of overlapping CKR profiles in defining fingerprints of specialized effector subsets, we next asked if functionally distinct subsets of $\mathrm{CXCR}^{+}$cells could be identified by the presence or absence of additional CKRs. Data presented in Figure 6 show that most Th1 cells that express CCR5, CCR2, CCR6, or CXCR5 actually coexpress CXCR3. Thus, in the absence of CXCR3, neither CCR5, CCR2, CCR6, or CXCR5 expression is associated with a significant frequency of Th1 cells, and none of these receptors predicts Th1 cytokine production by itself. In fact, the percentage of CXCR $3^{+}$double-positive cells in these populations $\left(75 \%\right.$ in $\mathrm{CCR}^{+}, 73 \%$ in CCR2 $2^{+}, 48 \%$ in CCR $6^{+}$, $25 \%$ in $\mathrm{CXCR5}^{+}$) positively correlates with their polarity toward Th1 within each CKR-defined subset. More importantly, the data lead to interesting and surprising conclusions about the significance of coexpression of 
each of these receptors with CXCR3. CCR5 and CCR6, respectively, have only marginal positive and negative association with the frequency of Th1 or Th2 cells among the $\mathrm{CXCR} 3^{+}$population, suggesting that these receptors are more likely regulated independently of Th1 cytokine preference (Figure 6, a and c). On the other hand, expression of CCR 2 is associated with a decrease rather than an increase in Th1 frequency and a slight increase in Th 2 cells among CXCR $3^{+}$cells (Figure 6b). CXCR5 is most strongly associated with a decrease in Th1 frequency (Figure 6d).

\section{Discussion}

Recent literature has led to two widely cited theses regarding the association of CKR expression and lymphocyte homing properties with $\mathrm{T}$ cell polarization or effector function. The first thesis is that some CKRs may be exclusively expressed by either Th1 or Th 2 cells; the second is that $\mathrm{CCR} 7^{+}$memory $\mathrm{T}$ cells are defective in effector function, while CCR7- $\mathrm{T}$ cells comprise the immediate effector pool. In this study, CKR expression by in vivo polarized Th 0 and Tnp cells was examined in three different but complementary ways: analyses of polarized and nonpolarized cell frequency in each CKR-defined subset; direct examination of CKR expression by polarized effector (Th1 and Th2), Th0, and Tnp memory CD4 T cell subsets; and analyses of combinatorial CKR expression by polarized cells. Our results call into question current assumptions and reveal novel features of CKR expression by polarized effector and nonpolarized memory CD4 $\mathrm{T}$ subsets that imply the capacity for differential targeting of these populations in the setting of diverse homing programs.
In the context of differential CKR expression, our results support two major conclusions: first, that none of the CKRs studied here is exclusively expressed by Th1, Th2, Th0, or Tnp cells, and second, that a number of CKR-defined subsets are nonetheless relatively enriched in their content of Th 1 or Th 2 cells. Our results show that there are several classes of Th1- or Th2-associated CKRs. The first is expressed by almost all Th 1 cells or all Th2 cells (CXCR3 for Th1 and CCR4 for Th2). The second class of CKRs is only expressed by subset of Th1 or Th2 cells, yet is preferentially displayed by the cells of one effector type: CCR2, CCR5, and CXCR6 for Th1 are in this group. None of the CKRs studied fall into this group for Th2 cells in our hands including CCR3. In this regard, other scientists have reported CCR3 is not reproducibly expressed on Th2 cells $(26,27)$. Yet another class of CKRs, including CXCR5, CCR7, and CCR6, show no preferential association and indeed are negatively related to $\mathrm{T}$ cell polarization: $\mathrm{T}$ cells expressing these CKRs, especially CXCR5, contain a reduced frequency of polarized $\mathrm{T}$ cells compared with the total memory $\mathrm{T}$ cell population.

Recent reports of enhanced frequency of Th $1 / 2$ cells among blood CCR7- $\mathrm{T}$ cells have led to the erroneous conclusion that CCR7 and polarized $\mathrm{T}$ cell functions are mutually exclusive; i.e., $\mathrm{CCR} 7^{+} \mathrm{T}$ cells are defective in immediate effector function and home exclusively to secondary lymphoid tissues, while effector cytokineproducing cells are CCR7 ${ }^{-}$and home only to nonlymphoid tissues (42). Our results demonstrate that, actually, there are many more CCR $7^{+}$than CCR7- Th1 and Th2 cells in blood. Expression of CCR7 may be necessary for targeting $\mathrm{CCR}^{+} \mathrm{T}$ cells into lymphoid tissues,
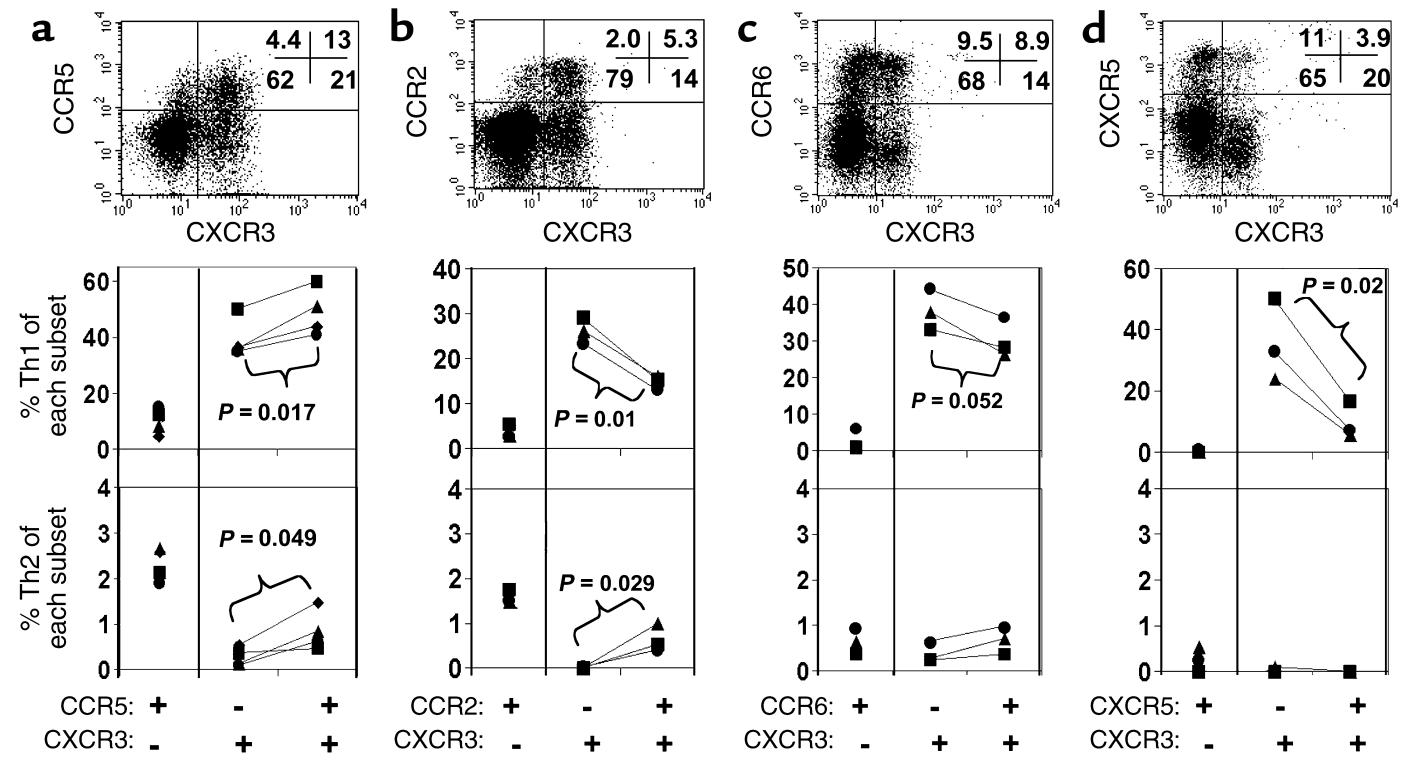

Figure 6

Dependence of Th1-containing populations on coexpression of CXCR3. Peripheral blood CD4 T cells expressing CXCR3 and/or another CKR among CCR5 (a), CCR2 (b), CCR6 (c), or CXCR5 (d) are shown along with frequencies of Th1 and Th2 cells. Each subset was sorted after staining with Ab's to CKRs, stimulated with PMA and ionomycin, and examined for production of IL-4 and/or IFN- $\gamma$. Results from three to four donors are shown. 
but is not sufficient to prevent the homing of polarized $\mathrm{CCR}^{+} \mathrm{T}$ cells into nonlymphoid tissues. Indeed, CCR7 ${ }^{+}$ memory $\mathrm{T}$ cells express other CKRs such as CXCR3 and CCR4 that can target cells to nonlymphoid tissues (data not shown). Thus, many polarized CCR $7^{+} \mathrm{Th} 1$ or Th2 cells may be able to home to lymphoid organs as well as extralymphoid tissues. In line with this, Randolph et al. have reported that in vitro-generated Th1 cells express CCR7 and migrated well into $\mathrm{T}$ cell areas of the spleen (48). Homing of polarized T cells into lymphoid tissues may lead to amplification of Th1 or Th2 cell generation from naive $\mathrm{T}$ cells. It has been reported also that CCR7 does not discriminate IFN- $\gamma$ producers from non-IFN- $\gamma$ producers among CD8 T cells (49).

Our results show that many Tnp's that are unable to produce IFN- $\gamma$ or IL-4 can also express a number of (nonlymphoid tissue homing) CKRs such as CCR5, CXCR3, and/or CCR4 in addition to lymphoid homing-related receptors CCR7 and CXCR5. These findings suggest that these $\mathrm{T}$ cells also have a homing potential to nonlymphoid tissues and may have other functions other than effector cytokine production. This is supported by the fact that significant portions of $\mathrm{T}$ cells isolated from various nonlymphoid tissues are defective in Th1 or Th2 cytokine production, and in some tissue sites many infiltrating memory phenotype $\mathrm{T}$ cells are $\mathrm{CCR} 7^{+}$(Figure 5 and ref. 50).

Although our results show that CCR 4 is expressed by most Th 2 cells and CXCR 3 by most Th 1 cells in circulation, we describe significant numbers of CCR $4^{+} \mathrm{Th} 1$ and $\mathrm{CXCR}^{+} \mathrm{Th} 2$ cells, most of which are contained among a $\mathrm{CXCR} 3^{+} \mathrm{CCR} 4^{+}$(dual receptor expressing) memory CD4 population. Thus, a strong and specific correlation with Th1 and Th2 subsets requires simultaneous evaluation of both receptors: $\mathrm{CXCR} 3^{+} \mathrm{CCR} 4^{-}$ and CCR $4^{+}$CXCR $3^{-}$Th cells comprise Th 1 and Th2 cells, respectively, whereas CXCR $3^{+} \mathrm{CCR} 4^{+}$cells contain relatively more Th0 cells and significant (if reduced) frequencies of both Th1 and Th 2 cells, as well. These findings raise the possibility that distinctive combinations of receptors may confer specialized homing behavior on these major effector cell populations. Identification of the CXCR $3^{+} \mathrm{CCR} 4^{+}$subset may also help us explain the apparently contradictory roles proposed for CCR4: $\mathrm{CLA}^{+}$skin homing $\mathrm{T}$ cells, which are almost all $\mathrm{CCR} 4^{\text {high }}$, contain a high frequency of $\mathrm{CXCR} 3^{+} \mathrm{CCR} 4^{+}$ cells. Therefore, association of CCR 4 with skin-homing $\mathrm{T}$ cells means that not only Th2 $\left(\mathrm{CLA}^{+} \mathrm{CCR} 4^{+} \mathrm{CXCR} 3^{-}\right)$ cells, but also Th1, Th0 $\left(\mathrm{CLA}^{+} \mathrm{CCR} 4^{+} \mathrm{CXCR}^{+}\right)$, and Tnp cells are able to home to skin. In fact, the heterogeneity of skin-homing cells should permit each different effector $\mathrm{T}$ cell subset to home to skin in normal and diseased conditions.

The CKR expression pattern by the CD4 T cells from arthritic synovium is different from that of circulating blood CD4 T cells in that they are enriched with T cells coexpressing tissue homing-related CKRs such as CXCR3, CCR5, CXCR6, and CCR4. Particularly, they are enriched for $\mathrm{CXCR} 3^{+} \mathrm{CCR} 4^{+}$double-positive $\mathrm{T}$ cells with an enhanced frequency of Th0 cells and fewer polarized (Th1 or Th2) cells than single-positive populations expressing either CXCR3 or CCR4. These data are well supported by the fact that, despite being $T$ cells from a Th1-oriented disease, the frequency of IFN- $\gamma$ producers among the Th1-oriented $\mathrm{CKR}^{+}\left(\mathrm{CCR}^{+}, \mathrm{CXCR}^{+}\right.$, and CXCR $6^{+}$) synovial fluid CD4 $\mathrm{T}$ cells are actually lower than those of their blood counterparts. Furthermore, the Th0 frequency (3-4\%) of these tissue CD4 T cells is higher (1.1\%) than that of blood memory CD4 T cells. Therefore, we conclude that the rules of CKR association with $\mathrm{T}$ cell polarization, initially discovered for peripheral blood CD4 $\mathrm{T}$ cells (Figures 1-4), apply also to the tissue CD4 T cells (Figure 5). Expression of the lymphoid-homing CKR CCR7 by many polarized and nonpolarized $\mathrm{T}$ cells in arthritic synovium further confirms that CCR7 expression by itself should not be regarded as an indicator for lack of effector function or for homing phenotype only to lymphoid tissues.

Our findings also suggest an explanation for the preferential expression of CCR 5 or CCR 2 by many Th 1 cells. These CKRs are most often coexpressed with CXCR3 in blood and some tissues, and in fact the smaller subsets of CCR5 and CCR2 cells that lack CXCR 3 contain few Th1 (and more Th2) cells. Thus these CKRs may be considered "opportunistic Th1associated markers," since their association with Th1 polarization appears to depend largely on coexpression with of CXCR3 (Figure 6).

CXCR3 ligands such as IP-10, MIG, and I-TAC, and CCR4 ligands such as MDC and TARC, are expressed by a number of cell types and are widely associated with sites of inflammation (51-54). These CXCR3 and CCR4 ligands differ in regulation because the CXCR3 ligands are induced by IFN- $\gamma$, while the CCR4 ligands are induced by IL-4 and/or IL-13 in cell types including macrophages, endothelial cells, and bronchial epithelial cells. Thus, our results suggest the potential for differential targeting of major populations of Th1 and Th2 cells based on their differential expression of these two receptors and their chemokine ligands: in this model, CXCR3 would act in concert with other coexpressed receptors (including, in some instances, CCR7, more often CCR5 and CCR2, and even CCR4 in inflamed skin) in targeting of Th1 cells and CCR 4 in concert with others (including CXCR3 to inflamed sites and CCR7 to lymphoid tissues) in targeting of Th2 cells.

In conclusion, our results demonstrate, in contrast to previous reports, that polarized effector and nonpolarized memory $\mathrm{T}$ cells in blood and tissues display diverse CKRs and thus suggest that they are highly heterogeneous in trafficking properties. However, this heterogeneity is not random but follows certain trends. Thus the heterogeneity does not exclude the possibility that major populations of Th1, Th2, and Tnp cells are targeted differentially in vivo, because successful navigation and differential targeting of cells require the simultaneous display of multiple receptors (in unique combinations) on the cell surface (5). 


\section{Acknowledgments}

We thank J.Y. Kim, M. Williams, and D.J. Campbell for critical comments on this paper. This work is supported by grants from the NIH, an award from the Department of Veterans Affairs (E.C. Butcher), and fellowships from the Leukemia and Lymphoma Society (C.H. Kim) and the Arthritis Foundation (E.J. Kunkel).

1. Cyster, J.G. 1999. Chemokines and cell migration in secondary lymphoid organs. Science. 286:2098-2102.

2. Butcher, E.C., Williams, M., Youngman, K., Rott, L., and Briskin, M. 1999. Lymphocyte trafficking and regional immunity. Adv. Immunol. 72:209-253.

3. Kim, C.H., and Broxmeyer, H.E. 1999. Chemokines: signal lamps for trafficking of T and B cells for development and effector function. J. Leukoc. Biol. 65:6-15.

4. Sallusto, F., Mackay, C.R., and Lanzavecchia, A. 2000. The role of chemokine receptors in primary, effector, and memory immune responses. Annu. Rev. Immunol. 18:593-620.

5. Foxman, E.F., Campbell, J.J., and Butcher, E.C. 1997. Multistep navigation and the combinatorial control of leukocyte chemotaxis. J. Cell Biol. 139:1349-1360.

6. Abbas, A.K., Murphy, K.M., and Sher, A. 1996. Functional diversity of helper T lymphocytes. Nature. 383:787-793.

7. Murphy, K.M., et al. 2000. Signaling and transcription in T helper development. Annu. Rev. Immunol. 18:451-494.

8. O'Garra, A. 2000. Commitment factors for T helper cells. Curr. Biol. 10:R492-R494.

9. Breitfeld, D., et al. 2000. Follicular B helper T cells express CXC chemokine receptor 5 , localize to B cell follicles, and support immunoglobulin production. J. Exp. Med. 192:1545-1552.

10. Schaerli, P., et al. 2000. CXC chemokine receptor 5 expression defines follicular homing $\mathrm{T}$ cells with B cell helper function. J. Exp. Med. 192:1553-1562.

11. Iezzi, G., Scheidegger, D., and Lanzavecchia, A. 2001. Migration and function of antigen-primed nonpolarized T lymphocytes in vivo. J. Exp. Med. 193:987-994

12. Kim, C.H., Campbell, D.J., and Butcher, E.C. 2001. Nonpolarized memory $\mathrm{T}$ cells. Trends Immunol. 22:527-530.

13. Bonecchi, R., et al. 1998. Differential expression of chemokine receptors and chemotactic responsiveness of type $1 \mathrm{~T}$ helper cells (Th1s) and Th2s. J. Exp. Med. 187:129-134.

14. D'Ambrosio, D., et al. 1998. Selective up-regulation of chemokine receptors CCR4 and CCR8 upon activation of polarized human type 2 Th cells. J. Immunol. 161:5111-5115.

15. Loetscher, P., et al. 1998. CCR5 is characteristic of Th1 lymphocytes. Nature. 391:344-345.

16. Sallusto, F., Lenig, D., Mackay, C.R., and Lanzavecchia, A. 1998. Flexible programs of chemokine receptor expression on human polarized $\mathrm{T}$ helper 1 and 2 lymphocytes. J. Exp. Med. 187:875-883.

17. Sallusto, F., Mackay, C.R., and Lanzavecchia, A. 1997. Selective expression of the eotaxin receptor CCR3 by human T helper 2 cells. Science. 277:2005-2007.

18. Zingoni, A., et al. 1998. The chemokine receptor CCR8 is preferentially expressed in Th2 but not Th1 cells. J. Immunol. 161:547-551.

19. Kim, C.H., et al. 2001. Bonzo/CXCR6 defines polarized type $1 \mathrm{~T}$ cell subsets with extra-lymphoid tissue homing potential. J. Clin. Invest. 107:595-601.

20. Austrup, F., et al. 1997. P- and E-selectin mediate recruitment of T-helper1 but not T-helper- 2 cells into inflammed tissues. Nature. 385:81-83.

21. Lim, Y.C., et al. 1999. Expression of functional selectin ligands on Th cells is differentially regulated by IL-12 and IL-4. J. Immunol. 162:3193-3201.

22. Andrew, D.P., et al. 2001. CCR4 expression defines a major subset of circulating non-intestinal memory $\mathrm{T}$ cells of both Th1 and Th2 potential. $J$. Immunol. 166:103-111.

23. Yamamoto, J., et al. 2000. Differential expression of the chemokine receptors by the Th1- and Th2-type effector populations within circulating CD4+ T cells. J. Leukoc. Biol. 68:568-574.

24. Nanki, T., and Lipsky, P.E. 2000. Lack of correlation between chemokine receptor and $\mathrm{T}(\mathrm{h}) 1 / \mathrm{T}(\mathrm{h}) 2$ cytokine expression by individual memory $\mathrm{T}$ cells. Int. Immunol. 12:1659-1667.

25. Annunziato, F., et al. 1999. Assessment of chemokine receptor expression by human Th1 and Th2 cells in vitro and in vivo. J. Leukoc. Biol. 65:691-699.

26. Imai, T., et al. 1999. Selective recruitment of CCR4-bearing Th2 cells toward antigen-presenting cells by the $\mathrm{CC}$ chemokines thymus and activation-regulated chemokine and macrophage-derived chemokine. Int. Immunol. 11:81-88.

27. Romagnani, P., et al. 1999. Tryptase-chymase double-positive human mast cells express the eotaxin receptor CCR3 and are attracted by CCR3-binding chemokines. Am. J. Pathol. 155:1195-1204.
28. Charo, I.F., et al. 1994. Molecular cloning and functional expression of two monocyte chemoattractant protein 1 receptors reveals alternative splicing of the carboxyl-terminal tails. Proc. Natl. Acad. Sci. USA. 91:2752-2756.

29. Daugherty, B.L., et al. 1996. Cloning, expression, and characterization of the human eosinophil eotaxin receptor. J. Exp. Med. 183:2349-2354.

30. Ponath, P.D., et al. 1996. Molecular cloning and characterization of a human eotaxin receptor expressed selectively on eosinophils. J. Exp. Med. 183:2437-2448

31. Kitaura, M., et al. 1996. Molecular cloning of human eotaxin, an eosinophil-selective CC chemokine, and identification of a specific eosinophil eotaxin receptor, CC chemokine receptor 3. J. Biol. Chem. 271:7725-7730.

32. Imai, T., et al. 1997. The T cell-directed CC chemokine TARC is a highly specific biological ligand for CC chemokine receptor 4. J. Biol. Chem. 272:15036-15042

33. Combadiere, C., Ahuja, S.K., Tiffany, H.L., and Murphy, P.M. 1996. Cloning and functional expression of CC CKR5, a human monocyte CC chemokine receptor selective for MIP-1(alpha), MIP-1(beta), and RANTES. J. Leukoc. Biol. 60:147-152.

34. Raport, C.J., Gosling, J., Schweickart, V.L., Gray, P.W., and Charo, I.F. 1996. Molecular cloning and functional characterization of a novel human CC chemokine receptor (CCR5) for RANTES, MIP-1beta, and MIP-1alpha. J Biol. Chem. 271:17161-17166.

35. Baba, M., et al. 1997. Identification of CCR6, the specific receptor for a novel lymphocyte-directed CC chemokine LARC. J. Biol. Chem. 272:14893-14898.

36. Greaves, D.R., et al. 1997. CCR6, a CC chemokine receptor that interacts with macrophage inflammatory protein 3alpha and is highly expressed in human dendritic cells. J. Exp. Med. 186:837-844.

37. Fitzhugh, D.J., Naik, S., Caughman, S.W., and Hwang, S.T. 2000. cutting edge: C-C chemokine receptor 6 is essential for arrest of a subset memory Tcells on activated dermal microvascular endothelial cells under physiologic flow conditions in vitro. J. Immunol. 165:6677-6681.

38. Loetscher, M., et al. 1996. Chemokine receptor specific for IP10 and mig: structure, function, and expression in activated T-lymphocytes. J. Exp. Med. 184:963-969.

39. Forster, R., Emrich, T., Kremmer, E., and Lipp, M. 1994. Expression of the G-protein-coupled receptor BLR1 defines mature, recirculating B cells and a subset of T-helper memory cells. Blood. 84:830-840.

40. Matloubian, M., David, A., Engel, S., Ryan, J.E., and Cyster, J.G. 2000. A transmembrane CXC chemokine is a ligand for HIV-coreceptor Bonzo. Nat. Immunol. 1:298-304.

41. Unutmaz, D., et al. 2000. The primate lentiviral receptor Bonzo/STRL33 is coordinately regulated with CCR5 and its expression pattern is conserved between human and mouse. J. Immunol. 165:3284-3292.

42. Sallusto, F., Lenig, D., Forster, R., Lipp, M., and Lanzavecchia, A. 1999. Two subsets of memory $\mathrm{T}$ lymphocytes with distinct homing potentials and effector functions. Nature. 401:708-712.

43. Zabel, B.A., et al. 1999. Human G protein-coupled receptor GPR-9-6/CC chemokine receptor 9 is selectively expressed on intestinal homing $\mathrm{T}$ lymphocytes, mucosal lymphocytes, and thymocytes and is required for thymus-expressed chemokine-mediated chemotaxis. J. Exp. Med. 190:1241-1256.

44. Zaballos, A., Gutierrez, J., Varona, R., Ardavin, C., and Marquez, G. 1999. Cutting edge: identification of the orphan chemokine receptor GPR-9-6 as CCR9, the receptor for the chemokine TECK. J. Immunol. 162:5671-5675.

45. Youn, B.S., Kim, C.H., Smith, F.O., and Broxmeyer, H.E. 1999. TECK, an efficacious chemoattractant for human thymocytes, uses GPR-9-6/CCR9 as a specific receptor. Blood. 94:2533-2536.

46. Campbell,J.J., et al. 1999. The chemokine receptor CCR4 in vascular recognition by cutaneous but not intestinal memory $\mathrm{T}$ cells. Nature. 400:776-780.

47. Christophers, E. 2001. Psoriasis: epidemiology and clinical spectrum. Clin Exp. Dermatol. 26:314-320.

48. Randolph, D.A., Huang, G., Carruthers, C.J., Bromley, L.E., and Chaplin, D.D. 1999. The role of CCR7 in TH1 and TH2 cell localization and delivery of B cell help in vivo. Science. 286:2159-2162.

49. Champagne, P., et al. 2001. Skewed maturation of memory HIV-specific CD8 T lymphocytes. Nature. 410:106-111.

50. Campbell, J.J., et al. 2001. CCR7 expression and memory $\mathrm{T}$ cell diversity in humans. J. Immunol. 166:877-884.

51. Farber, J.M. 1997. Mig and IP-10: CXC chemokines that target lymphocytes. J. Leukoc. Biol. 61:246-257.

52. Sauty, A., et al. 1999. The T cell-specific CXC chemokines IP-10, Mig, and I-TAC are expressed by activated human bronchial epithelial cells. $J$. Immunol. 162:3549-3558.

53. Andrew, D.P., et al. 1998. STCP-1 (MDC) CC chemokine acts specifically on chronically activated Th2 lymphocytes and is produced by monocytes on stimulation with Th2 cytokines IL-4 and IL-13. J. Immunol. 161:5027-5038.

54. Sekiya, T., et al. 2000. Inducible expression of a Th2-type CC chemokine thymus- and activation-regulated chemokine by human bronchial epithelial cells. J. Immunol. 165:2205-2213. 